\title{
The role of the Medical Devices Agency
}

Most doctors are familiar with the Medicines Control Agency (MCA) and their 'yellow card system' for reporting possible adverse events involving drugs but fewer are aware of the Medical Devices Agency (MDA) and its role in the safety of medical devices.

The term medical device incorporates a huge range of healthcare products from single-use items used every day (such as syringes, needles and dressings) to large hospitalbased equipment (such as x-ray machines). Contraceptives are classed as either devices or medicines depending on their mode of action. A contraceptive which is classed as a medical device is one which does not achieve its principal intended action by pharmacological, immunological or metabolic means but which may be assisted in its function by such means. Oral contraceptives are classed as medicines, as are depot injections. Things are not so clear when it comes to intrauterine devices (IUDs); IUDs containing copper are classified as devices while those releasing hormones are medicines.

How a contraceptive is classified will depend upon the claims made by the manufacturer. So a contraceptive sponge containing a spermicide may be classified as either a medicine or a device. If the manufacturer claims that the presence of the sponge is the main contraceptive action and the spermicide acts as a secondary function then the product will be classified as a device. If the manufacturer claims that the main contraception action is that of the spermicide with the sponge acting as a delivery system then the product will be classified as a medicine.

So why does it matter how a contraceptive is classified? Medical devices and medicines are regulated and controlled using two very different systems. Medicines are licensed under the Medicines Act and European Medicinal Products Directive and manufacturers have to obtain a product licence from the MCA or European Medicines Evaluation Agency (EMEA) before they can be sold in the UK. Conversely, medical devices are regulated using the European Medical Devices Directive 93/42/EEC, and before a contraceptive device can be marketed it needs to be accredited by a independent third party (known as a Notified Body) and be CE marked. Those who want to know more can read all about the regulations on MDA's website or contact us for more details (see overleaf).

MDA is responsible for ensuring the safety and quality of all medical devices used in the UK. We rely on doctors, nurses and other healthcare workers within hospitals and the community to make reports of adverse events to MDA's Adverse Incident Centre (AIC). Adverse incidents may arise from shortcomings in a device, its operating instructions, user practices, servicing and maintenance or conditions of use. The importance of an incident is not always obvious, and types of incidents vary from extremely serious to apparently minor. What may seem to be a minor problem can take on greater significance when aggregated with other similar events.

In 2001 MDA received nearly 8000 reports of problems involving a broad range of medical devices; of these 41 involved IUDs. Investigation may show that the incident was an isolated one and under such circumstances no further action is needed. However, it could also reveal that there is a problem with a particular batch of products, the product design or the instructions for use.

When a problem has been identified MDA can do several things. In the most extreme cases we can take enforcement action to prevent the product's further use. However this rarely happens. More commonly we work with the manufacturer to correct the problem, inform users and recall products were necessary.

MDA issues advice to the healthcare providers in the form of Hazard Notices or Device Alerts (specific advice for immediate action) or Safety Notices (information which users need to avoid a potential hazard). In 2001 MDA issued 6 Hazard Notices, 10 Device Alerts and 35 Safety Notices. A full list of the notices issued can be found on our website.

In the past year reports to MDA of problems involving two different types of IUDs from UK doctors/nurses have led to in-depth investigations by MDA. These resulted in changes in the designs of both IUDs, intended to make insertion easier and also in one case reduce the incidence of perforations. In these cases the UK supplier of the IUDs sent out letters to all their customers informing them of the changes; MDA is continuing to monitor these devices closely. Reports received in 1999 of the blue ring stoppage collar/depth marker moving on the Gyne-T380 IUD introducer led to MDA issuing a Safety Notice, MDA SN 1999(22), to warn users of the problem and give advice about what action should be taken.

MDA is keen to hear about all events that cause, or have the potential to cause, unexpected or unwanted effects involving the safety of patients, users or others. This would include all serious events even if the event is a known complication such as an IUD perforating the uterus or pregnancy following IUD fitting. It is important to monitor the rates of known complications such as these because an increase may suggest a problem with a particular batch or model of device. Incidents of a less serious nature should also be reported.

Adverse incidents can be reported online by simply filling out an adverse incident report form, available on our website, or by calling +44 (0) 2079728080 for a hard copy. You can also write to us at AIC, MDA, Hannibal House, Elephant and Castle, London SE1 6TQ, UK, or fax us on +44 (0) 207972 8109. If you are unsure of whether a problem should be reported you can always give us a call on the above telephone number.

If you would like to find out more about MDA and its work, information can be found on our website at www.medical-devices.gov.uk.

Statements on funding and competing interests

Funding. None declared.

Competing interests. None declared.

Alison Austin, $\mathrm{PhD}$
Senior Device Specialist, Implants and Materials, MDA,
Hannibal House, Elephant and Castle, London SE1 6TQ,
UK
$U K$ 